\title{
Terapia medica della sindrome di Cushing: aggiornamento su relacorilant e levoketoconazolo
}

\author{
Alessia Cozzolino ${ }^{1}$. Marianna Minnetti ${ }^{1}$. Valeria Hasenmajer ${ }^{1}$. Valentina Sada ${ }^{1}$. Dario De Alcubierre ${ }^{1}$. \\ Riccardo Pofi ${ }^{1}$. Emilia Sbardella ${ }^{1}$. Andrea Lenzi ${ }^{1}$. Andrea M. Isidori ${ }^{1}$
}

Accettato: 3 dicembre 2020 / Pubblicato online: 18 marzo 2021

(c) The Author(s) 2021

La sindrome di Cushing è una malattia endocrina rara associata a molteplici complicanze sistemiche e a un'aumentata mortalità, quando è trattata in maniera subottimale [1]. La terapia medica rappresenta un'opzione per i pazienti non candidati alla neurochirurgia o per i quali questa non sia stata risolutiva. I farmaci attualmente disponibili si dividono in tre categorie: farmaci diretti all'ipofisi, inibitori della steroidogenesi surrenalica e antagonisti del recettore dei glucocorticoidi. Nell'ultimo decennio ci sono stati nuovi sviluppi in ognuna delle tre categorie [1].

\section{Relacorilant}

Relacorilant è un antagonista del recettore dei glucocorticoidi altamente selettivo, attivo per via orale, che modula gli effetti del cortisolo in eccesso senza mostrare alcuna affinità per i recettori del progesterone, dei mineralcorticoidi, degli androgeni o degli estrogeni (Fig. 1). A differenza del mifepristone, infatti, relacorilant non si lega al recettore del progesterone ed è pertanto privo degli effetti antiprogestinici, come l'interruzione di gravidanza, l'ipertrofia endometriale e il sanguinamento vaginale irregolare [2]. In uno studio condotto per la prima volta sull'uomo in volontari sani, il farmaco è stato ben tollerato dopo dosi ripetute fino a $250 \mathrm{mg}$ una volta al giorno per 14 giorni con un buon profilo di sicurezza e gli effetti anti-glucocorticoidi sono stati confermati [2]. In uno studio di fase 2 che ha arruolato 35 pazienti con sindrome di Cushing, il tratta-

$凶$ A. Cozzolino

alessia.cozzolino@hotmail.com

1 Dipartimento di Medicina Sperimentale, Sapienza Università di Roma, Roma, Italia<smiles>Cn1cc(S(=O)(=O)N2CCC3=Cc4c(cnn4-c4ccc(F)cc4)CC3(C(=O)c3cc(C(F)(F)F)ccn3)CC2)cn1</smiles>

Relacorilant<smiles>COC#C[C@]1(O)CC[C@H]2[C@H]3CCC4=CC(=O)CCC4=C3[C@@H](c3ccc(N(C)S(C)(=O)=O)cc3)C[C@@]21C</smiles>

Mifepristone
Fig. 1 Struttura chimica di relacorilant e mifepristone. Il relacorilant presenta nella sua struttura chimica fluoro (F) e zolfo (S) non presenti nel mifepristone

mento con relacorilant ha mostrato un miglioramento degli endpoint glicemici e ipertensivi. I pazienti arruolati venivano divisi in due coorti, sulla base della dose di farmaco: gruppo basse dosi (100-200 mg/die) e gruppo alte dosi (250-400 mg/die). Dopo 4 settimane di trattamento, relacorilant ha indotto un miglioramento dei livelli pressori nel 41,7 e nel $63,6 \%$ dei pazienti, con un miglioramento nel metabolismo glucidico nel 15,4 e nel 50\% dei pazienti, nei due gruppi rispettivamente [3]. Relacorilant è stato ben tollerato, senza casi di ipopotassiemia correlata al farmaco o effetti antiprogestinici.

Attualmente, è in corso uno studio clinico multicentrico di fase 3 randomizzato (studio GRACE: NCT03697109) per valutare l'efficacia e la sicurezza del relacorilant nel trattamento dei pazienti con sindrome di Cushing. Lo studio è mirato a obiettivi di efficacia clinica, in particolare il miglioramento delle alterazioni del metabolismo glucidico e dell'ipertensione arteriosa, confrontando il trattamento con relacorilant e quello con placebo [4]. 
Fig. 2 Struttura chimica di levoketoconazolo e ketoconazolo. Il levoketoconazolo è l'enantiomero $2 \mathrm{~S}, 4 \mathrm{R}$ del ketoconazolo

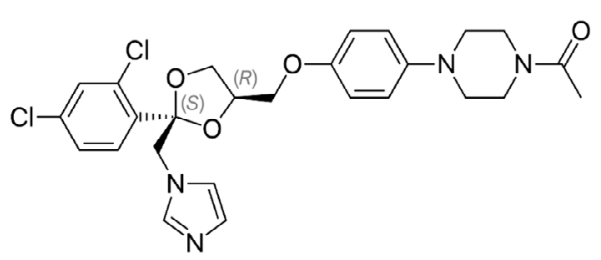

Levoketoconazolo

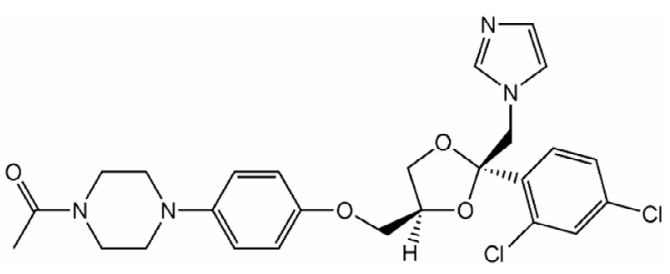

Ketoconazolo

\section{Levoketoconazolo}

Il levoketoconazolo è l'enantiomero $2 \mathrm{~S}, 4 \mathrm{R}$ del ketoconazolo (Fig. 2). Analogamente al ketoconazolo racemico, il levoketoconazolo inibisce CYP11B1, CYP17 e CYP21, ma in modo più potente, e questo potrebbe consentire l'utilizzo di dosi inferiori di farmaco. Al contrario, il levoketoconazolo ha un effetto inibitorio più debole sul CYP7A epatico, la cui ridotta attività può determinare lo sviluppo di una colestasi funzionale. Le suddette caratteristiche rendono il levoketoconazolo potenzialmente meno epatotossico del ketoconazolo racemico. Inoltre, rispetto al ketoconazolo, il levoketoconazolo ha un migliore profilo farmacocinetico [1]. Il farmaco è stato inizialmente studiato in soggetti con diabete mellito di tipo 2, trattati con dosi crescenti, con una buona tollerabilità, salvo per un incremento dose-dipendente del numero di disturbi gastrointestinali e cefalea [5].

Recentemente, lo studio SONICS di fase 3, multicentrico, open-label, non randomizzato ha valutato l'efficacia e la sicurezza del trattamento con levoketoconazolo in pazienti con sindrome di Cushing. L'outcome primario era rappresentato dalla proporzione di pazienti che aveva ottenuto la normalizzazione del cortisolo libero urinario medio al termine della fase di mantenimento, senza aver incrementato la dose di farmaco. Lo studio ha arruolato 94 pazienti e di questi 29 (31\%) hanno raggiunto l'outcome primario. Sono stati inoltre osservati miglioramenti significativi dei marcatori delle principali comorbidità della sindrome di Cushing: glicemia a digiuno, HbA1c, colesterolo totale e LDL e peso corporeo. Sono stati riportati significativi miglioramenti di acne e irsutismo nelle donne, oltre che dell'edema periferico, e un miglioramento della qualità della vita $(\mathrm{Cu}-$ shing QoL) e della severità della depressione (valutata secondo il BDI-II score). Relativamente al profilo di sicurez$\mathrm{za}$, il farmaco ha mostrato una buona tollerabilità. Gli eventi avversi più frequenti sono stati la nausea $(32 \%)$ e la cefalea $(28 \%)$. Gli eventi avversi seri più frequenti sono stati l'ipertensione e l'incremento dei livelli circolanti di alanina aminotransferasi (ALT) [6].

Funding Note Open access funding provided by Università degli Studi di Roma La Sapienza within the CRUI-CARE Agreement.
Conflitto di interesse Gli autori Alessia Cozzolino, Marianna Minnetti, Valeria Hasenmajer, Valentina Sada, Dario De Alcubierre, Riccardo Pofi, Emilia Sbardella, Andrea Lenzi e Andrea M. Isidori dichiarano di non avere conflitti di interesse.

Consenso informato Lo studio presentato in questo articolo non ha richiesto sperimentazione umana.

Studi sugli animali Gli autori di questo articolo non hanno eseguito studi sugli animali.

Nota della casa editrice Springer Nature rimane neutrale in riguardo alle rivendicazioni giurisdizionali nelle mappe pubblicate e nelle affiliazioni istituzionali.

Open Access This article is licensed under a Creative Commons Attribution 4.0 International License, which permits use, sharing, adaptation, distribution and reproduction in any medium or format, as long as you give appropriate credit to the original author(s) and the source, provide a link to the Creative Commons licence, and indicate if changes were made. The images or other third party material in this article are included in the article's Creative Commons licence, unless indicated otherwise in a credit line to the material. If material is not included in the article's Creative Commons licence and your intended use is not permitted by statutory regulation or exceeds the permitted use, you will need to obtain permission directly from the copyright holder. To view a copy of this licence, visit http://creativecommons.org/licenses/by/4.0/.

\section{Bibliografia}

1. Feelders RA, Newell-Price J, Pivonello R et al (2019) Advances in the medical treatment of Cushing's syndrome. Lancet Diabetes Endocrinol 7:300-312

2. Hunt H, Donaldson K, Strem M et al (2018) Assessment of safety, tolerability, pharmacokinetics, and pharmacological effect of orally administered CORT125134: an adaptive, double-blind, randomized, placebo-controlled phase 1 clinical study. Clin Pharm Drug Dev 7(4):408-421

3. Pivonello R, Kargi AY, Ellison N et al (2019) Efficacy and safety of the selective glucocorticoid receptor modulator, relacorilant (up to $400 \mathrm{mg} /$ day), in patients with endogenous hypercortisolism: results from an open-label phase 2 study. American Association of Clinical Endocrinologists Annual Congress 2019

4. Auchus R, Findling J, Moraitis A (2019) A randomizedwithdrawal, placebo-controlled, phase 3 study to assess the efficacy and safety of selective glucocorticoid receptor antagonist, relacorilant, in patients with Cushing syndrome (GRACE Study). J Endocr Soc 3(Suppl 1):451 
5. Schwartz SL, Rendell M, Ahmann AJ et al (2008) Safety profile and metabolic effects of 14 days of treatment with DIO-902: results of a phase IIa multicenter, randomized, double-blind, placebocontrolled, parallel-group trial in patients with type 2 diabetes mellitus. Clin Ther 30:1081-1088
6. Fleseriu M, Pivonello R, Elenkova A et al (2019) Efficacy and safety of levoketoconazole in the treatment of endogenous $\mathrm{Cu}$ shing's syndrome (SONICS): a phase 3, multicentre, open-label, single-arm trial. Lancet Diabetes Endocrinol 7:855-865 